\title{
A Tentative Theory of Human's Thoughts and Behaviors
}

\author{
K. Wang,*
}

\begin{abstract}
This paper presents a new idea to understand human's thoughts and behaviors. Here, we propose a tentative theory of human's thoughts and behaviors, including the relativity of the thoughts, the relativity of the behaviors with thoughts, the inertia of the behaviors with thoughts and its corollary, the spiral advance of the human's thoughts, the procedure of the behaviors with thoughts, and a universal identity. This paper aims to provide a new method to know ourselves and lead human's life better. We design some experiments to check the theory lastly.
\end{abstract}

Keywords: thoughts, behaviors, time, speed of thinking, movement.

Introduction- One difficult thing for people is to know themselves, especially their thoughts. As we all know, the thoughts is so hard to detect that the most useful way to measure our thoughts is our feelings. Although some bioscientists are trying to measure human's brain waves to understand human's thoughts $[1,2]$, they have seen little to date. Since the human is very complex, finding a universal theory to explain the thoughts and furthermore, the relation between the thoughts and the behaviors is very difficult, and even impossible to achieve in decades. In this letter, we try to provide a new way to understand human's thoughts and behaviors. It is an intersection of physics, psychology, philosophy and human's behaviors. However, due to the difficulty of experiment, we just give a qualitative explain.

This letter includes six parts. Firstly, we discuss the relativity of the thoughts, which is the most important and fundamental in this paper. Secondly, we discuss the ideological behavior theory. Thirdly, we discuss the inertia of the behaviors with thoughts and its corollary. Fourthly, we discuss the spiral advance of the thoughts. Fifthly, we discuss the procedure of the behaviors with thoughts. Lastly, we give a universal identity, which can be explained for everything, but also just qualitatively.

The Relativity of The thoughts-Firstly, people have subjective initiative and can perceive time. Thus, there exists a thinking time and space, called the Internal Time and Space (ITS), contrast to the External Time and Space (ETS). We propose the following theory: When people are actively thinking, the faster their thinking speed of the Internal Time and Space (TSITS), the slower the time of Internal Time and Space (TITS), the faster the time of the External Time and Space (TETS), and vice versa; when people are passive thinking, the faster their TSITS, the faster the TITS, the slower the TETS, and vice versa. The so-called "actively thinking" refers to the thinking when our brain is awake, and we can control our thoughts, such as thinking at work or exams, thinking when people are playing with the electronic devices and etc. In contrast, the so-called "passive thinking" refers to the thinking when our brain is in a coma state, and we are not free to control our thoughts, such as thinking when people are asleep, called "dream", thinking when people are in a coma or lethargy state and etc.
The meaning of ITS: Human live in three-dimensional space and one-dimensional time. So the human belong to three-dimenisional biological. However, the thoughts of people are some different. Human are human because they have thoughts, they are conscious. The consciousness exists in our brains, but it has no entity. The consciousness belongs to an independent time and space, that is ITS. The ITS consists of one-dimensional time and one-dimensional space, both can not be seen by us. But we can feel them, and they are related to our body and interact with each other. And when our brains die, they will disappear because there is no carrier any more.

Let $V$ be the TSITS, which is a function of human's states, especially human's brain, even related to every neurons. Let $\Delta t_{I}$ be the TITS and $\Delta t_{E}$ be the TETS. Let $C$ be the maximum of $V$, which means that the human's thinking speed has an extreme value, for everybody. Different people may have different maximum $C$. Maybe when your entire brain's neurons are working at the fastest speed, your thinking speed can reach the maximum, but you can't reach this level with the current level of human's intelligence, or you may never reach it, it's just an ideal value. Like the speed of light in nature, unless the mass of object is 0 , it will never reach the speed of light. Next, we discuss the "actively thinking" condition. According to the description of the theory, we know that the theory satisfies the following requirements: When $V$ is equal to 0 , the rate $\frac{\Delta t_{I}}{\Delta t_{E}}$ should be infinity, because in this case, people can be considered dead; When $V$ is equal to a value $V_{0}$, the rate $\frac{\Delta t_{I}}{\Delta t_{E}}$ is 1 . In this case, the speed of human's internal and external time is equal; When $V$ reaches the maximum $C$, the rate $\frac{\Delta t_{I}}{\Delta t_{E}}$ should be 0 . And in this case, it is no exaggeration to say that the human beings are eternal. Based on these three requirements, we make the following formula for qualitative assumptions.

$$
\frac{\Delta t_{I}}{\Delta t_{E}}=\Phi(V)=\frac{V_{0}}{V} \sqrt{\frac{C^{2}-V^{2}}{C^{2}-V_{0}^{2}}} .
$$

For the "passive thinking" condition, we simply count down the $\Phi(V), \frac{\Delta t_{I}}{\Delta t_{E}}=\frac{1}{\Phi(V)}$, and the function can satisfy the description of the theory.

Another question is how to measure the TSITS and TITS. This is a very difficult problem. Because this kind 

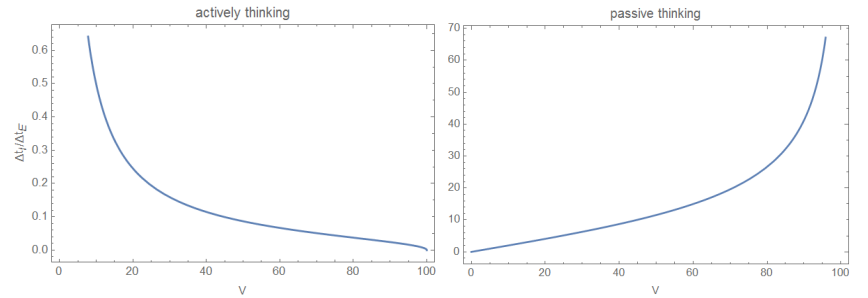

FIG. 1: A qualitative description. In order to facilitate the study of changing laws, we take $V_{0}=5, C=100$ for example. The abscissa is $V$, and the ordinate is the rate $\frac{\Delta t_{I}}{\Delta t_{E}}$. The left is "actively thinking" condition and the right "passive thinking" condition.

of measurement can't be done by outsiders, unless ourselves, but our own measurements are not necessarily accurate and objective. Therefore, we know, a more correct theory will gradually emerge with the development of science and technology. And we hope that our theory is the beginning of this field, more people will do reseach in this area in the future. So far, we take the idea that the amount of information that appears in the brain per unit time can be used as the TSITS. This may be measured by measuring the brain waves of human-brain thinking in experiments. However, the TITS is really difficult to measure because we can not put a clock in our internal space and time, the only way we can use now is the feelings of people themselves.

We now take some examples to explain: If you are burying yourself in a math text or reading a novel seriously, or thinking about a scene quickly, when the outside world has been in the past two hours, then you may only feel that it has been one hour, because your TSITS is faster than $V_{0}$.

If you have ten minutes left in class, you have nothing to do, there is only one thought in your thoughts, "Hurry up, let's get out of class!" and often look your watch, then after class, maybe you thought it was half an hour's length because your TSITS is slower than $V_{0}$.

For the case of being actively accepting outside information, it is also the first category (actively thinking). For example, when watching TV, the brain constantly inputs images and texts from the screen, and they enter the brain to form "brain memory", which is equivalent to increasing their thinking speed, and if you are looking at videos that are not of interest, then information can't be input into your brain and form "brain memory," which of course doesn't increase your thinking speed.

When you have a nap for a while, you can't control your thoughts and you are in "passive thinking". If you have a lot of scenes in your thoughts like dreaming one after another, and the performance is very fast, then when you wake up, you think that you have passed two hours, but in fact only half an hour later. However, when you sleep at night, if you don't have many dreams, then ex-

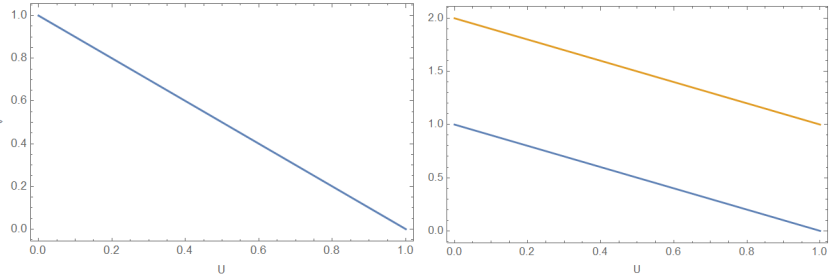

FIG. 2: A qualitative description for the relation of thoughts and behaviors. In order to facilitate the study of changing laws, we take $V_{\max }=1, U_{\max }=1$ for example. The abscissa is the speed of behaviors $U$, and the ordinate is the speed of thinking $V$. The left is represent that people is in a common state and the right represent the lift of the state. For example, the person secretes more adrenaline.

ternal eight or nine hours are just like internal an hour or two hours.

Interestingly, in this way, people's lifespan can not just look at the surface, because there is an inner time dimension, which is the more real lifespan experience in fact.

The Ideological Behaviors Theory-In unit time, When people is in a certain state, which means that the energy consumed by him in a unit of time is certain, the speed of human's behaviors (SHB) is inversely related to the TSITS. In other words, the higher the SHB (that is, the more you do in a certain period of time), the lower the TSITS. Conversely, the lower the SHB, the higher the TSITS. This relation can be described by the following qualitative formula,

$$
\frac{V}{V_{\max }}+\frac{U}{U_{\max }}=1 \text {. }
$$

When human's state is lifted, for example, the person secretes more adrenaline, then the values of $V_{\max }$ and $U_{\max }$ will get bigger. The corresponding figure is shown in Fig. (2).

For example, when you are doing violent exercise, your SHB will become larger, and your TSITS will become smaller (assuming that you do not deliberately improve your state to increase the TSITS), and then according to the previous theory-the relativity of the thoughts, when you feel that you have been for an hour, the outside world has only been half an hour. So, speaking less and doing more, you can think farther and deeper.

The Inertia of the Behaviors With Thoughts and Its Corollary-When a person suddenly remembers something else (not related to what he is doing, or does not take the initiative to change the current behaviors), and thinks about it with a high degree of concentration, then his body will remain in its original state, i.e., staying still or continue the current behaviors unless there are external conditions to change this state of thoughts, or outside conditions to block his behaviors, or his thoughts return to himself (i.e., wake up from contemplation). The higher the speed of thinking other things, the more stable the 
original state is, and the higher the conditions required to change the original state. This rule can be qualitatively described by the formula, $\Psi(V)=k V$, where $V$ is the speed of thinking other things, and $\Psi(V)$ represents the size of condition required to change the state of thoughts (or change its state of motion) under the corresponding $V$. The degree of $\Psi(V)$ is a relatively abstract concept, perhaps the intensity of the sound, the frequency of the sound, or the strength of the outside world to touch you. In short, it is the minimum degree of external conditions to change the original state.

On the other hand, changing the state of thoughts and changing the state of physical exercise are to some extent the same, because once the state of thoughts changes (such as the outside world pulls your meditation back into reality), it can often directly lead to changes in your body's state of motion, and the state of motion. The change of the state of physical exercise can often stimulate the change of state of thoughts, but the former change is relatively easier.

For example: When you are looking at a place (like watching a distant landscape), suddenly you think of a mathematical problem, and then desperately want to work out it, then you will maintain this state of the body, the eyes will not even blink, the body will not move. If your thinking speed is relatively slow, even if the sound of an embroidery needle falling to the ground can "awake" you; but if your thinking speed is relatively fast, then even someone call you behind, you will not notice that.

The Corollary-Under the same external conditions, the faster the thinking speed, the smaller the influence of external conditions on it, the slower the thinking speed, and the greater the influence of external conditions on it.

For example, when a person is doing something, if he is fully engaged, and his thinking speed is high, the external environment will hardly affect him, even if you call his name or pat him, he may not know.

The Spiral Advance of the Thoughts-The development of the human's thoughts is spiral, it will change with the length of life and the complexity of life, but it does not rise in a straight line in the process of change, it rises spirally and will alternate the emergence of "peaks" and "low valleys" during the ascent. Although fluctuating throughout the process, it is generally on the rise.

If there is a curve that can represent the development of people's thoughts, then their curves are different for different people: some may be slower and then faster, some may be faster and then slower, but overall, it is on the rise and spiraling upwards. And the development of thoughts presents a kind of periodicity.

Give an example of being close to life: even without outside interference, our energy, vitality, excitement, etc, are sometimes high, and sometimes low (and even pessimistic). In fact, the former is at the highest point of a spiral ring of the spiral curve, while the latter is at the lowest point. This feeling will alternate and will not last a sense without change. Here is actually the realm of thoughts to be specific, or to turn it into a human's feelings. This is ok, because the spiral forward model not only adapts to the abstract concept of the ideological realm, it also applies to things closer to humans (such as feelings, etc.), and the level of emotion is in fact unable to reflect the level of ideological realm. People's power, vitality, energy, excitement and many other "human parameters" (some standards that reflect the state of the human body) all satisfy the spiral forward curve of thought, and the ideological realm is actually a comprehensive reflection of all "human parameters". Of course, it is more in line with the spiral of thoughts.

The Procedure of the Behaviors With Thoughts-If a person is doing something, and he is already familiar with the process of doing it, then even if he shifts most of his attention away, leaving little attention to it, then his this behavior will still work, as if an behavior program had been set up in his mind.

At first glance, this law seems to be somewhat similar to the inertia of the previous thoughts and behaviors, but the two are different. The previous rule is that there is no need to keep in thoughts the process of behaviors, and this rule does not require a high speed of thoughts. Another point is that the previous law of inertia often maintains relatively simple behaviors such as simple swinging legs, walking or standing still, and the attention at that time is completely removed, and the behaviors allowed by this law are a bit more complicated than the former, but it is not very complicated, otherwise it may go wrong.

Regarding the biological cause of this phenomenon, I have a guess: when an (or a series of) behavior is repeated many times, its behavioral process will form a clear "loop" in the brain or the neurons are connected more closer by synapass, and pressing the "switch" from any position in this "loop" will successfully complete the rest, and even basically does not require you to actively intervene. In other words, this is like a gradual transformation from conditional reflex to unconditioned reflex. At first, the behavioral process is familiarized by learning behavior, and then you no longer need to intervene. The behavior is completed under a series of subconscious commands. The "loop" in the brain is equivalent to the behavioral program.

For example, if a person is carrying a box (not too heavy, it is easy to move it), hold it from the top of the car, then take a distance, put it in a designated position, repeat this work, when after he did it about a dozen times, his thought about a behavioral procedure in the subconscious thoughts (in fact, the process of writing this program was not human intervention), so even if he was absent-thoughtsed, he took most of his attention to other things, only very little attention is paid to this behavior, then in the end he can still do the job well, and at the end he will feel "what hasn't happened yet." This is actually because when he carries most of the boxes, only 
the behavioral program is working, and not the active consciousness.

A Universal Identity-This law can be seen as a universal philosophy of life.

$$
\hat{1}+\check{1}=\overline{2},
$$

where $\hat{1}$ and $\check{1}$ represent different things, including everything, respectively. The Eq. (3) means that the reduction of one thing is necessarily accompanied by the increase of another. This is a qualitative expression because many things can not be specifically represented by its quantity. Note that $\hat{1}$ and $\check{1}$ here can change at different and it is allowed that another change after a change.

In physics, the unified field theory may be born in the future. It uses equations to quantitatively describe the natural world. But it can't involve human's thoughts. The law of conservation of energy tells us that the total energy of the universe is certain. There will be an unified theoretical system in various fields (including physics, philosophy, life, mathematics, etc.), and the ultimate degeneration of each theory is the universal identity. Here, the principle of human existence is also important, because judgments like this really need people to think about it. Here are a few examples in different areas so that you can have a better understanding of this balancing principle and know how to apply it to solve problems.

First of all, the field of philosophy. In fact, there is a similar expression in philosophy: "You may get something when you lose something, and you may lose something when you get something." This sentence is simple and incisive. If we have "getting" in one aspect, we will inevitably have "missing" in another aspect. The arrangement in the universe is fair. But the philosophical statement is basically limited to the getting or losing specific things, the human's emotions are less. So the principle of balance is magnified here, it is not only applicable to the former field, but it can also be applied to the more abstract areas including human's emotions, the entire life and even the entire human race.

If a person fails an exam, this is a "loss", $\hat{1}$ is a reduced state, then the other $\check{1}$ will inevitably show an increase, which may occur before or after the "loss". Supposing that it happened before, then his "getting" may be spiritual satisfying behaviors, such as excessive relaxation and learning less, so that he gets lost in the exam and reaches a balance. For another example, if you often play sports for physical activities, your physical fitness is improved, and you get health. As the saying goes, paying is directly proportional to the harvest. The great people who have a lot of viewers, their lives are often more difficult, just to see how hard they work, we can know that they will lose a lot. Therefore, in theory, we should not envy anyone, or do not think that the great men are perfect, know that their struggle process is full of hardships, and that must be a process that will be lost a lot. The judgment of "gain" and "missing" should be a judgment made objectively.

Natural science such as physics and mathematics, this substitution, the principle of balance (3) is equally suitable. For example, in the process of nuclear fusion or nuclear fission, the quality of the system is reduced, so there must be something that has increased. Now there is an answer. The reduced mass of matter is converted into energy and released, and it conforms to the mass equation $E=m c^{2}$, which is given by Einstein. In the special theory of relativity, when the speed of an object increases, there must be something that is reduced. Now we know that the time of the object is slower than the outside, the length of the object will be shorter. But how the specific changes will require physicists to study, this formula can only give a qualitative explanation, or as a concept of life. Therefore, getting bigger and getting smaller is always one after another.

Thus, in the natural sciences, if we have a worldview of the principle of balance, it will help us discover new things and even new laws. When we find that something is decreasing (or increasing), we can explore what is increasing (or decreasing) of things.

Experimental Methods-Since this theory is applicable to human beings, the theory of verification can be done by randomly selecting 500 or more honest people to do experiments, reading them some text to calculate the speed of thinking per unit time, and then over a period of time, let them say that they have thought how long they have passed, take this time to compare with the actual time, and repeat the experiment, and finally the statistical average is used to verify the law. This method is used to test the relativity of the thoughts, and the remaining rules can also use the similar method to verify. [1-4]

Summary-The purpose of this paper is to connect human's thoughts and behaviors, to connect human's inner time and space with the external time and space, to link non-quantitative life and quantitative natural sciences, and to help people discover new things better and understand themselves in a new way.

*wk813943178@gmail.com

[1] B. J. Baars and N. M. Gage, Cognition, Brain, and Consciousness: Introduction to Cognitive Neuroscience (Academic Press; 2nd edition, 2010).

[2] B. Libet, Mind Time: The Temporal Factor in Consciousness (Perspectives in Cognitive Neuroscience) (Harvard University Press, 2005).

[3] S. Menon, Brain, Self and Consciousness (Springer India, 2014).

[4] D. Radin, Entangled Minds: Extrasensory Experiences in a Quantum Reality (Paraview Pocket Books; 45834th edition, 2006). 